\title{
A Model Dynamics of Interacting Firms
}

\author{
Naoyuki Ishimura and Masaaki Nakamura
}

\begin{abstract}
We deal with a system of nonlinear ordinary differential equations (ODEs), which models the dynamics of "immortality" of interacting companies. The system possesses blowing-up solutions. We determine the asymptotic behavior of these blowing-up solutions, and discuss the meaning in financial economics.
\end{abstract}

Index Terms-Asymptotic behavior, blowing-up solutions, dynamics of interacting companies, system of nonlinear ordinary differential equations.

\section{INTRODUCTION}

This paper is concerned with the following system of nonlinear ordinary differential equations (ODEs), which models the dynamics of interacting companies

$$
\begin{gathered}
u^{\prime}(t)=\alpha u(t)-\alpha v(t) w(t) \\
v^{\prime}(t)=\beta v(t)-\beta w(t) u(t) \\
w^{\prime}(t)=\gamma w(t)-\gamma u(t) v(t)
\end{gathered}
$$

where $\alpha, \beta, \gamma$ are positive constants. Unknown functions $u(t), v(t), w(t)$ represent the "immortality" of respective firms. The derivation of the system (1) will be recalled in Section II.

As is easily observed, the system (1) possesses blowing-up solutions in the sense that

$$
u(t)^{2} \rightarrow \infty, \quad v(t)^{2} \rightarrow \infty, \quad w(t)^{2} \rightarrow \infty \text { as } t \rightarrow T
$$

for some positive $T$. See Section III below. These behaviors are interpreted as representing that the firms may be defaulted and/or be renovated within finite period. The meaning of this observation will be considered later.

The aim of this paper is to determine the asymptotic behavior, in particular, the rate of blowing-up for these solutions.

Our main theorem reads as follows:

Theorem. For every sufficiently large initial data $\left(u_{0}, v_{0}, w_{0}\right)$ in the sense that $u_{0}^{2}, v_{0}^{2}, w_{0}^{2}$ are large enough with $u_{0} v_{0} w_{0}<0$, the solution beaks down within finite time.

Conversely, if the solution $(u(t), v(t), w(t))$ to (1) blow-up as $t \rightarrow T$, then we have

Manuscript received October 19, 2012; revised January 14, 2013. This work was supported in part by the grants of Japan Society for Promotion of Sciences (JSPS) (Nos. 21540117, 21540147). The first author (N.I.) is also supported in part by G-COE Hi-Stat Program Hitotsubashi University.

Naoyuki Ishimura is with the Graduate School of Economics, Hitotsubashi University, Tokyo, Japan (e-mail: ishimura@ econ.hit-u.ac.jp).

MasaAki Nakamura is with the College of Science and Technology, Nihon University, Kanda-Surugadai, Tokyo, Japan (e-mail: nakamura@math.cst.nihon-u.ac.jp).

$$
\begin{aligned}
& \lim _{t \rightarrow T} \sqrt{\beta \gamma}(T-t) u(t)=: u_{*} \\
& \lim _{t \rightarrow T} \sqrt{\gamma \alpha}(T-t) v(t)=: v_{*}, \\
& \lim _{t \rightarrow T} \sqrt{\alpha \beta}(T-t) w(t)=: w_{*}
\end{aligned}
$$

with $\left(u_{*}\right)^{2}=\left(v_{*}\right)^{2}=\left(w_{*}\right)^{2}=-u_{*} v_{*} \mathrm{~W}_{*}=1$.

Moreover if we define $x(t), y(t)$ and $z(t)$ by

$$
\begin{gathered}
u(t)=(T-t)^{-1} u_{*}(1+x(t)) / \sqrt{\beta \gamma}, \\
v(t)=(T-t)^{-1} v_{*}(1+y(t)) / \sqrt{\gamma \alpha}, \\
w(t)=(T-t)^{-1} w_{*}(1+z(t)) / \sqrt{\alpha \beta},
\end{gathered}
$$

then it follows that

$$
\begin{gathered}
\lim _{t \rightarrow T}(\beta+\gamma-\alpha)^{-1}(T-t)^{-1} x(t)=1 / 2, \\
\lim _{t \rightarrow T}(\gamma+\alpha-\beta)^{-1}(T-t)^{-1} y(t)=1 / 2, \\
\lim _{t \rightarrow T}(\alpha+\beta-\gamma)^{-1}(T-t)^{-1} z(t)=1 / 2
\end{gathered}
$$

That is, the second order behavior of blowing-up solutions is established. Furthermore, we note that the possible choices of $\left(u_{*}, v_{*}, w_{*}\right)$ are

$$
(-1,-1,-1),(-1,1,1),(1,-1,1),(1,1,-1) .
$$

Numerical study is performed in Section 4 and the implications in financial economics of this theorem will be discussed in Section V.

\section{MODEL}

Here we briefly recall the derivation of the system (1).

Suppose that the there are three firms whose "immortality" are represented by $X(t), Y(t), Z(t)$, respectively, where $t$ denotes the time variable. The immortality is just an abstract quantity. If $X(t)=-\infty$, then the company $X$ is interpreted as being defaulted. While $X(t)=\infty$, then $X$ is considered to be graded-up or ranked-up to the higher level.

We assume that the rate of immortality is divided into two parts; the one is the spontaneous growth part and the other is the exogenous depreciation part. The depreciation part is assumed to come from the interaction of other two firms only. The resulting system of ODEs now becomes

$$
\begin{aligned}
& X^{\prime}(t)=a X(t)-b Y(t) Z(t) \\
& Y^{\prime}(t)=c Y(t)-d Z(t) X(t) \\
& Z^{\prime}(t)=e Z(t)-f X(t) Y(t)
\end{aligned}
$$

where $a, b, c, d, e, f$ are positive constants. If we put

$$
\begin{aligned}
& u(t)=\sqrt{c^{-1} d e^{-1} f} X(t), \quad \alpha=a, \\
& v(t)=\sqrt{a^{-1} b e^{-1} f} Y(t), \quad \beta=b, \\
& w(t)=\sqrt{a^{-1} b c^{-1} d} Z(t), \quad \gamma=c,
\end{aligned}
$$


then we recover the system (1).

We remark that the change of variables $u \rightarrow-u, v \rightarrow-v$ and $w \rightarrow-w$ transforms the system (1) into

$$
\begin{aligned}
u^{\prime}(t) & =\alpha u(t)+\alpha v(t) w(t) \\
v^{\prime}(t) & =\beta v(t)+\beta w(t) u(t) \\
w^{\prime}(t) & =\gamma w(t)+\gamma u(t) v(t)
\end{aligned}
$$

The structure of solutions to (1) and (4) is therefore seen to be the same. Other possibility is

$$
\begin{aligned}
u^{\prime}(t) & =\alpha u(t)+\alpha v(t) w(t) \\
v^{\prime}(t) & =\beta v(t)+\beta w(t) u(t) \\
w^{\prime}(t) & =\gamma w(t)-\gamma u(t) v(t)
\end{aligned}
$$

and its variants. However, it is shown that every solution to (5) grows-up exponentially as the time tends to infinity; namely,

$u(t)^{2}+v(t)^{2}+w(t)^{2} \rightarrow \infty$ exponentially as $t \rightarrow \infty$.

Therefore, the structure of solutions to (5) and its variants is rather trivial. We will discuss the meaning of the system (1) further in Section 5.

\section{SKETCH OF PROOF}

Here we give a sketch of main theorem. The proof proceeds along the same line as in [1]. Indeed, if we make the reversion of the time $t \rightarrow-t$, then (1) reduces to

$$
\begin{aligned}
u^{\prime}(t) & =-\alpha u(t)+\alpha v(t) w(t) \\
v^{\prime}(t) & =-\beta v(t)+\beta w(t) u(t) \\
w^{\prime}(t) & =-\gamma w(t)+\gamma u(t) v(t)
\end{aligned}
$$

which is exactly the same system treated in [1]. Concerning the analysis below, we thus refer to [1] and we just provide the outline.

Proposition. The equilibrium points of (1) are

$(0,0,0),(1,1,1),(1,-1,-1),(-1,-1,1),(-1,1,-1)$.

The origin is unstable. Other four points have two dimensional stable manifold and one dimensional unstable manifold.

This proposition follows directly from the analysis on the linearized system of ODEs.

Proposition. For any solution of (1) whose initial value $\left(u_{0}, v_{0}, w_{0}\right)$ satisfies

$$
\begin{gathered}
u_{0} \ll-\max \{\alpha, \sqrt{\beta}, \sqrt{\gamma}\}, \\
v_{0} \ll-\max \{\sqrt{\alpha}, \beta, \sqrt{\gamma}\}, \\
w_{0} \ll-\max \{\sqrt{\alpha}, \sqrt{\beta}, \gamma\}
\end{gathered}
$$

breaks down within finite time.

This proposition is proved by the application of Gronwall's inequality. It is to be noted that the other combination of the sign for $(u, v, w)$ is also possible.

The rest of the proof is almost the repetition of those of [1]; we derive the differential equations for $(x(t), y(t), z(t))$ and establish various estimates, which we may safely omit the details.

\section{NUMERICAL STUDY}

We perform numerical implementation for (1). We refer to the related previous work of [2].

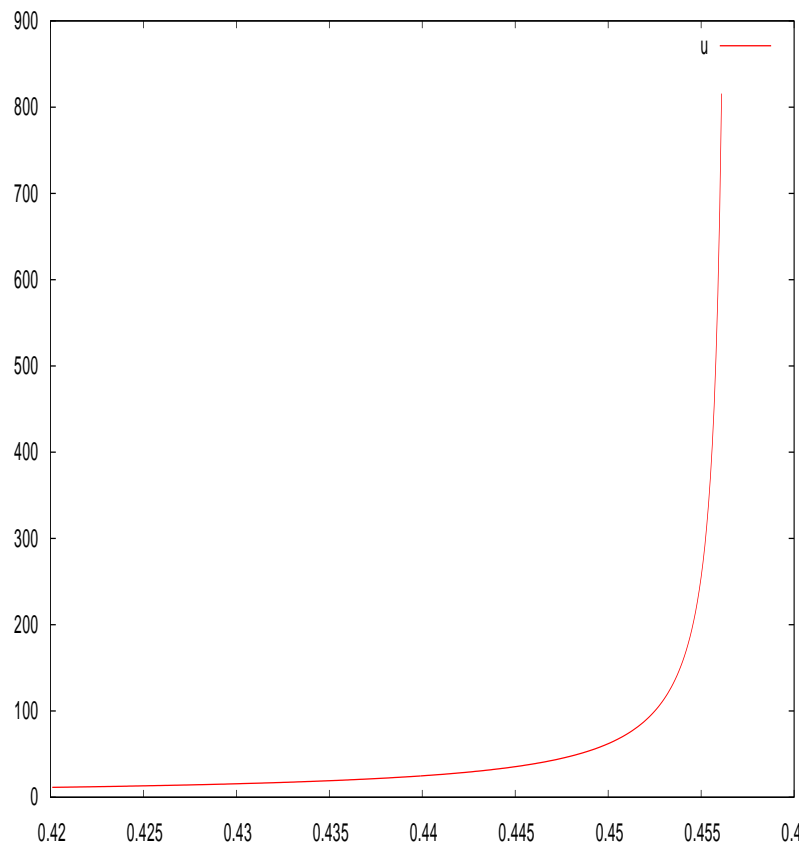

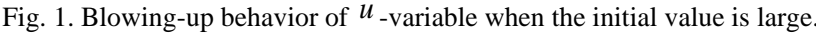

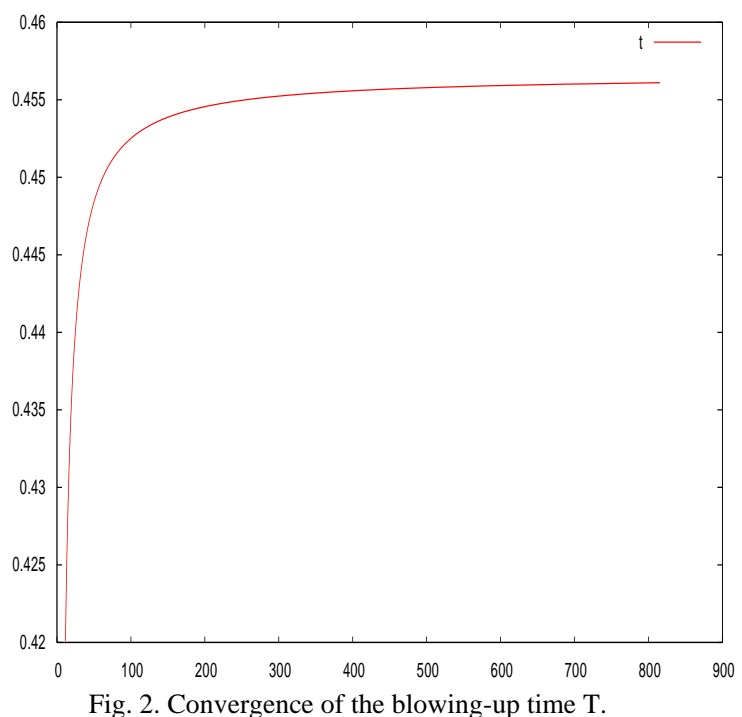

We use the 30-digits fourth order Runge-Kutta scheme to approximate the solutions. As is well known, the numerical computation for the blowing-up profiles is a little subtle. The careful treatment is therefore necessary.

The behaviors of each $u, v, w$-variables as well as the blowing-up time are exhibited in the following figures, where the constants are set to be $\alpha=4.0 \beta=3.0 \gamma=2.0$, which are the same as in [2].

Fig. 1 shows the blowing-up of the $u$-variable when the initial value is sufficiently large; we consider the sigh system so that the $u$-variable takes positive value.

The blowing-up patterns of the $v$ and $w$-variables are also 
similar. Of course the sign of the $v$ or $w$-variable should be negative.

Next, Fig. 2 shows the convergence of the blowing-up time with respect to the $u$-variable. The same profiles apply to the case of $v$ and $w$-variables since the blowing-up times are proved to be the same for all variables.

It can be seen that the blowing-up takes place drastically near the blowing-up time. Since the graph indicates the saturation, we understand that our computation scheme well works.

\section{DISCUSSION}

We have introduced a system of nonlinear ordinary differential equations (ODEs), which models the fate of interacting three firms. The governing system is obtained solely theoretically, where the company grows spontaneously but may fade because of the influence of other companies. Self-growth is assumed to be linear and of Malthus type, while the depreciation is considered to be nonlinear and of the simplest multiplicative components. These terms can be much complicated; however, such generalizations make the analysis much harder.

It is shown that firms can be defaulted and/or ranked-up within finite time. The pattern of these phenomena is interesting: One pattern is that all three companies fall in default simultaneously. The other is that one company is graded-up and the rest two companies are defaulted. Finally, two firms are ranked-up and the rest one firm is defaulted. We hope that these consequences may shed light on the analysis of existing firms.

On the other hand, the existence of equilibrium points of the system indicates that the firms may co-exist substantially stably. These occasions, however, are rather rare with respect to the initial conditions, which can be observed ubiquitously in the real world.

The innovative point of our main theorem is that the second order behavior of breaking down solutions is clarified. Although the technique employed there is elementary, the result is in a sense sharp. The rate of blowing-up is proved to be the same for all companies, which is natural from the viewpoint of the modeling of the interaction. The numerical computation of such blowing-up solutions, although the system is time-reversal, is also performed in our previous paper [1], where the good agreement with the theory is observed as well.

The mathematical models involving ODEs are very popular in applied sciences. The nonlinear terms usually give rise to typical reaction terms in the reaction-diffusion partial differential equations (PDEs) models. We refer to, for instance in the ODE settings, [3]-[5] and the references cited therein. We believe that such modeling is also possible as well as useful in the field of social sciences including financial mathematics. We thus hope that our paper may advance this possibility and our paper is worth publishing.

\section{ACKNOWLEDGMENT}

We are grateful to Professor Akira Mizutani at Gakushuin University for fruitful discussions and to Professor Ken'ichi Nagasaki at Chiba Institute of Technology for helpful comments. Thanks are also due to the referee for nice suggestions which helps in improving the manuscript.

\section{REFERENCES}

[1] N. Ishimura and M. A. Nakamura, "Blowing-up behavior of a system of nonlinear ODEs," J. Res. Inst. Sci. Tech., Nihon Univ.

[2] M. Inoue, N. Ishimura, and M. A. Nakamura, "Numerical study on the systems of nonlinear ordinary differential equations for default risk model," in Proc. IEEE 17th International Conference on Industrial Engineering and Engineering Management, 2010, pp. 1066-1069.

[3] R. M. May and W. J. Leonard, "Nonlinear aspects of competition between three species," SIAM J. Appl. Math., vol. 29, pp. 243-253, 1975 .

[4] W.-M. Ni, K. Suzuki, and I. Takagi, "The dynamics of a kinetic activator-inhibitor system," J. Differential Equations, vol. 299, pp. 426-465, 2006.

[5] S. Smale, "On the differential equations of species in competition," $J$. Math. Biology, vol. 3, pp. 5-7, 1976

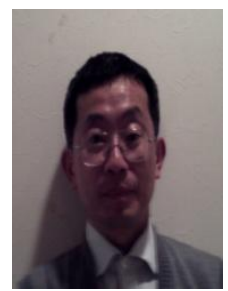

Naoyuki Ishimura graduated and obtained a PhD of Mathematical Sciences from the University of Tokyo, Japan. He is now Professor of Mathematical Finance at Graduate School of Economics, Hitotsubashi University, Tokyo, Japan. Prof Ishimura is a member of Japan Society of Industrial and Applied Mathematics (JSIAM).

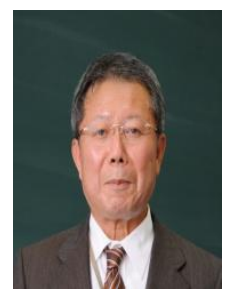

Masaaki Nakamura graduated and obtained a $\mathrm{PhD}$ of Mathematical Sciences from the University of Tokyo, Japan. He is now Professor of College of Science and Technology, Nihon University, Tokyo, Japan. Prof Nakamura is a member of Japan Society of Industrial and Applied Mathematics (JSIAM). 